\title{
POSTERIOR CEREBROVASCULAR ACCIDENTS AFTER PERCUTANEOUS CORONARY INTERVENTION
}

\author{
${ }^{1}$ Carlo Cosimo Quattrocchi, ${ }^{3}$ Andrea Maria Alexandre, ${ }^{1}$ Laura Scarciolla, ${ }^{2}$ Mario Tombini, \\ ${ }^{1}$ Eliodoro Faiella, ${ }^{1}$ Filomena Occhicone and ${ }^{1}$ Bruno Beomonte Zobel \\ ${ }^{1}$ Department of Radiology, Interdisciplinary Center for Biomedical Research, \\ ${ }^{2}$ Department of Neurology, Interdisciplinary Center for Biomedical Research, \\ University Campus Bio-Medico of Rome, Rome, Italy \\ ${ }^{3}$ Institute of Radiology, \\ Department of Bio-imaging and Radiological Sciences, Catholic University of Rome, Italy
}

Received 2012-05-31; Revised 2014-04-10; Accepted 2014-04-14

\begin{abstract}
Percutaneous Coronary Interventional procedures (PCIs) are recognised as gold standard treatment for high risks patients with diffuse coronary atherosclerosis. Neurologic complications are one of the most dreaded procedural outcomes, stroke after PCI affects mainly elderly and high-risk patients (age more than 80 years and use of intra-aortic balloon pump), with longer times of the procedure and angiographic complications, such as dissection, abrupt closure and no reflow. We report two consecutive cases of posterior CVAs after percutaneous coronary procedures involving the ponto-mesencephalon and the cerebellum. Especially when risk factors are present, careful attention should be devoted to the preparation of the patient and to minimize catheter manipulation and exchanges.
\end{abstract}

Keywords: Posterior Cerebrovascular Accidents, Percutaneous Coronary Interventions

\section{INTRODUCTION}

Percutaneous Coronary Interventional procedures (PCIs) are recognised as gold standard treatment for high risks patients with diffuse coronary atherosclerosis.

Neurologic complications are one of the most dreaded procedural outcomes, leading to an enormous impact on resource consumption, on patient's prognosis and quality of life, with a rate of reported stroke ranging from 0.11 to $0.4 \%$ (Fuchs et al., 2002).

Stroke after PCI affects mainly elderly and high-risk patients (age more than 80 years and use of intra-aortic balloon pump), with longer times of the procedure and angiographic complications, such as dissection, abrupt closure and no reflow (Fuchs et al., 2002).

Cerebrovascular Accidents (CVAs) complicating left heart catheterization procedures have been described most commonly in the fore part of the brain, as a consequence of embolic involvement of carotid supplied territories (Fuchs et al., 2002; Lund et al., 2005).

We report two consecutive cases of posterior CVAs after percutaneous coronary procedures involving the ponto-mesencephalon and the cerebellum.

\section{CASE REPORT}

\section{Case 1}

A 57-year-old woman, dislipidemic, with a two-year history of sudden and severe 10-min pre-cordial pain irradiated to the neck, jaw and to both upper limbs, spontaneously resolved.

The patient received ECG which revealed a mild mitral incontinence.

A 99Tc-Pyrophosphate myocardial scan demonstrated a reversible mild signal in the anterior descendent coronary artery territory.

Corresponding Author: Carlo Cosimo Quattrocchi, Department of Radiology, University Campus Bio-Medico of Rome, Via Longoni, 4700155 Rome, Italy Tel: 39-06-22541272 Fax: 39-06-22541456 
Admitted to the Cardiology Department she underwent diagnostic coronarography showing normal arteries.

The day after she suddenly developed left labial paresthesia, nausea, vomit and ocular disorders. She was oriented and alert. Neurological examination showed internuclear ophthalmoplegia, positive bilateral indexnose test, more evident on the right and mild postural tremor of the upper limbs.

MRI imaging of the brain at $48 \mathrm{~h}$ revealed small areas of high signal on diffusion weighted images in the right inferior quadrigeminal tubercle and in bilateral median and para-median portion of quadrigeminal lamina, a small left cerebellum infarct and small areas of high signal on T2-weighted and FLAIR images in the right subcortical and left fronto-parietal areas (Fig. 1a).

Ecocardiography and supra-aortic trunk ecocolordoppler showed no significant findings. The patient was managed conservatively.

At the 7 and 15 days follow-up MRI, blood-brain barrier disruption due to hemorrhagic reperfusion was observed as high signal intensity on T1-weighted images at the level of the lesions, in the right inferior quadrigeminal tubercle and in quadrigeminal lamina (Fig. $1 b$ and c).
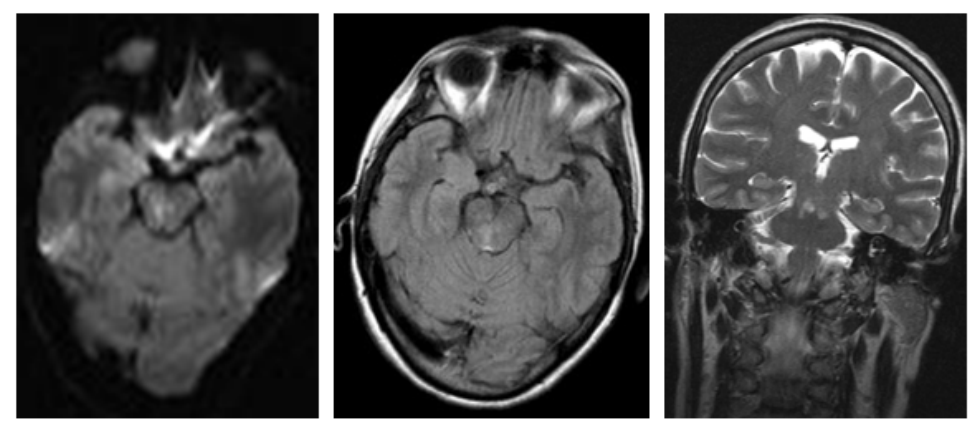

(a)
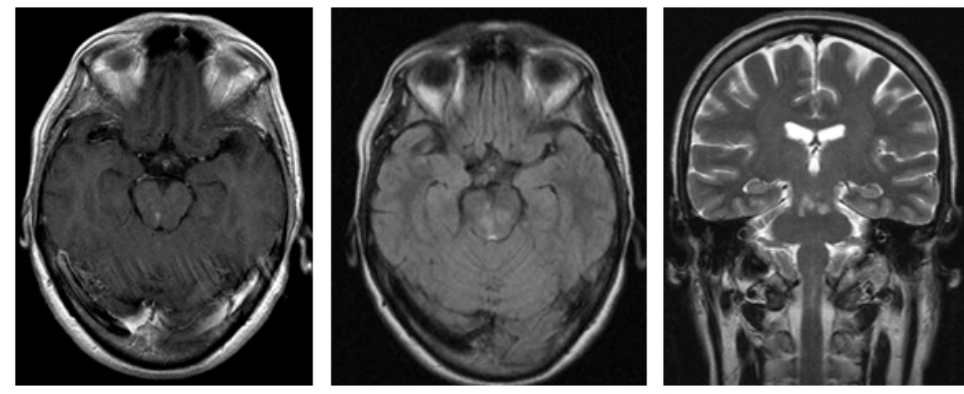

(b)
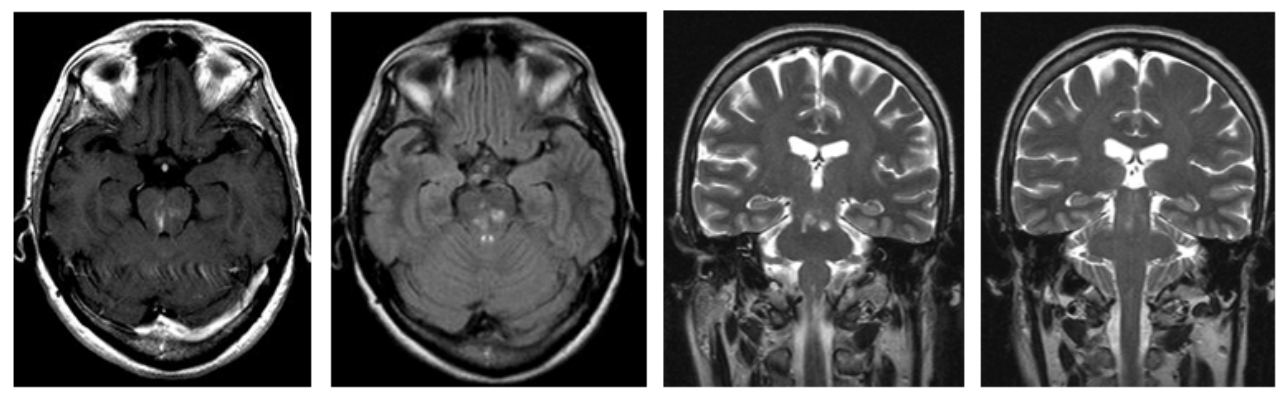

(c)

Fig. 1. MRI imaging of the brain at $48 \mathrm{~h}, 7$ days and 15 days after diagnostic coronarography (explanation in text): (a) Diffusion weighted image, axial FLAIR image and coronal T2-weighted images, (b) Axial T1-weighted image, axial FLAIR image and coronal T2-weighted images, (c) Axial T1-weighted image, axial FLAIR image and coronal T2-weighted images 


\section{Case 2}

A 57-year-old man, dyslipidemic, obese, with hypertension, ex-smoker, had positive history for recurrent anterior myocardial infarction treated with thrombolysis and with anterior descending artery stenting.

$\mathrm{He}$ developed unstable angina for which he was admitted to the Cardiology Department, after being asymptomatic for a period of 6 years.

Angiography showed total occlusion of the beginning of the right coronary artery, collateral circles and proliferation of intimal cells layers inside the stent along the anterior descending artery.

After the procedure, the patient complained dyspnea, nausea, vomit with a blood pressure of $140 / 70 \mathrm{mmHg}$, normal rate and blood oxygenation. He immediately started $\mathrm{O}_{2}$ therapy and Lasix $20 \mathrm{mg}$ ev.

Echocardiography showed left ventricle hypertrophy, aortosclerosis, moderate mitralic and tricuspid incontinence and moderate left atrium enlargement. Ventriculography showed normal size of the left ventricle but moderate anterolateral and postero-basal hypo kinesis and low ejection fraction (50\%).
Before discharging the patient, two events of angina were resolved with sublingual nitrates. Discharge therapy was: Cardioaspirin $100 \mathrm{mg}$, epinitril $10 \mathrm{mg}$, enalapril $20 \mathrm{mg}$.

Then he was admitted to our hospital because of a 36$\mathrm{h}$ loss of balance and co-ordination and 24-h lateral dyplopia and dysarthria appeared. The symptoms had been preceded by nausea and vomit 5 days earlier.

Neurological examination revealed horizontal lateral nystagmus (fast phase to the right), left adiadococinesia and impaired walking, suggesting internuclear ophthalmoplegia. Positive left index-nose test. Tone, strength and muscular trophism were preserved.

MRI imaging of the brain showed multiple areas of high signal on $\mathrm{T} 2$-weighted and FLAIR images involving the left paramedian ponto-mesencefalic region, the left cerebellar hemisphere and the right parietomesial region (Fig. 2a).

The high signal intensity on diffusion weighted images suggested subacute CVA in the vertebro-basilar territory. On the following MRI, 7 days later, the lesions were moderately enlarged and showed high signal intensity on T1-weighted images as for reperfusion blood-brain barrier damage (Fig. 2b).
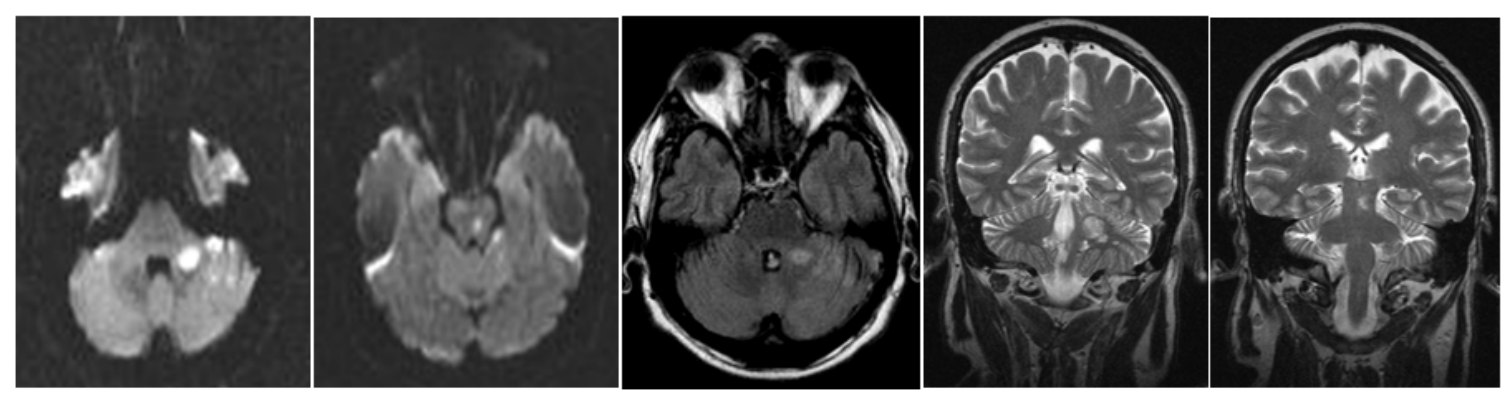

(a)
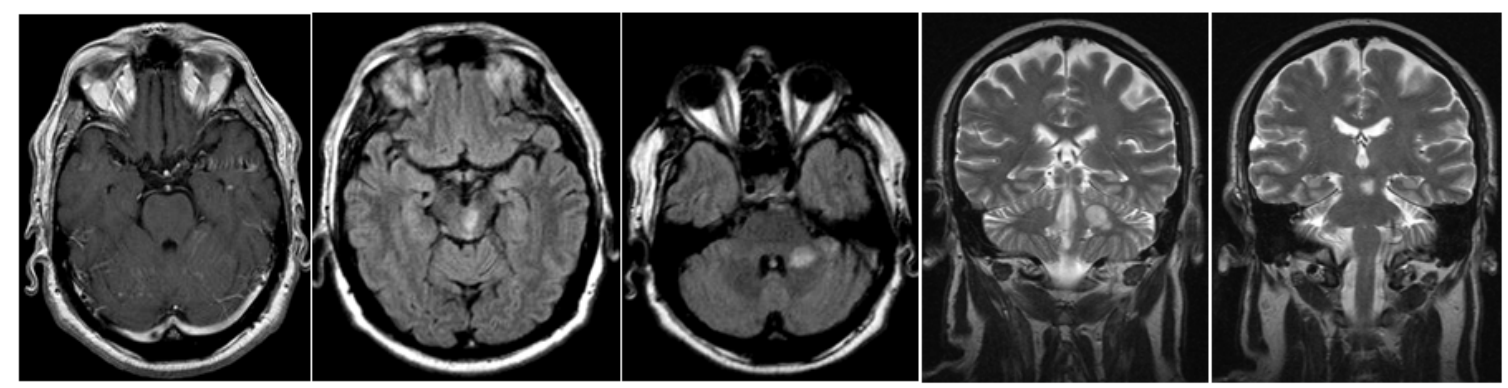

(b)

Fig. 2. MRI imaging of the brain at $48 \mathrm{~h}$ and 7 days after diagnostic coronarography (explanation in text): (a) Diffusion weighted images, axial FLAIR image and coronal T2-weighted images, (b) Post-contrast T1-weighted image, axial FLAIR images and coronal T2-weighted images 


\section{DISCUSSION}

Ischemic strokes most often involve the middle cerebral artery territory (up to 50\%) (Fuchs et al., 2002). Radiologic and postmortem studies indicate that perioperative strokes are predominantly ischemic and embolic. Ischemic stroke most often involve the major cerebral-arterial territories (middle cerebral artery, $47.6 \%$; posterior cerebral artery, $23.8 \%$; and anterior cerebral artery, $9.6 \%$ ).

The timing of embolic strokes after surgery has a bimodal distribution: $45 \%$ of perioperative strokes are identified within the first day after surgery, 55\% occur after recovery from anesthesia from the second postoperative day on. Early embolism results especially from manipulations of the heart and aorta. Lund et al. (2005; Dukkipati et al., 2005) found that brain lesions were associated with a significantly higher number of solid micro-emboli during transradial than during transfemoral catheterization.

The incidence of perioperative stroke depends on the type and complexity of the surgical procedure. The risk of stroke after general, noncardiac procedure is very low, whereas cardiac and vascular surgeries are associated with higher risk.

Fuchs et al. (2002) reported an incidence of stroke complicating PCI of $0.38 \%$ in 9,662 patients collected for 10 years. Recently Dukkipati et al. (2004) (Lund et al., 2005) published the largest single study, involving 20,679 patients collected for 9 years, with an event rate of $0.3 \%$.

The timing of procedure, total amount of contrast material or the type of contrast material are considered important factors to predict the risk.

Büsing et al. (2005) found that the rate of asymptomatic cerebral infarction after cardiac catheterization seems to be about 10 -fold higher than it is for clinically apparent cerebral lesions.

Other groups have recently documented that silent acute brain injury can also be associated with percutaneous cardiac interventions, with possible cognitive impairment for patients in whom new lesions are identified (Büsing et al., 2005; Dukkipati et al., 2004).

Even though the carotid system is probably more often involved, it is arguable that structures supplied by the vertebro-basilar system are more frequently symptomatic than those of the carotid system, because of low compensatory function in damaged cerebellum and brainstem and because of the absence of collateral circulation to the end-arteries supplying especially the brainstem.
Diffusion-Weighted Magnetic Resonance Imaging (DW-MRI), which provides image contrast based on random translational motion of water molecules, substantially altered by acute cerebral ischemia, is the most sensitive technique for early detection of cerebral infarction (Fiebach et al., 2001; Burdette et al., 1998).

The addition of DW-MRI to conventional sequences makes it possible to detect very small and hyperacute infarction at almost any anatomic location within the brain hemispheres, the brainsteam and the cerebellum, as shown in our cases. And structural damage to the brain has been detected by DW-MRI also in asymptomatic patients, caused by silent embolism during cerebral and coronary angiography (Hähnel et al., 2001; Bendszus et al., 1999; Omran et al., 2003), as well as during surgical and endovascular revascularization procedures in the carotid artery (Jaeger et al., 2001; Wityk et al., 2001).

Indipendent predictors for major periprocedural stroke have been shown to be: Age, sex, diabetes, history of hypertension, unstable angina, body weight, congestive heart failure, peripheral vascular disease, chronic renal dysfunction, history of transient ischemic attacks, vein graft intervention, use of abciximab. Fuchs et al (2002) showed that stroke after PCI affects mainly elderly patient with risk factors for atherosclerosis. Cerebral hemodynamic impairment in these patients would increase the risk of ischemic disease secondary to reduced flow (Weiller et al., 1991; Miyazava et al., 2001), which may be additionally compromised by systemic hypoperfusion.

Aortic atherosclerosis is an independent predictor of the risk of perioperative stroke: The use of ecocardiography-guided aortic cannulation and intra-aortic filtration can reduce the risk of stroke (Omran et al., 2003; Jackson et al., 2000).

Systolic dysfunction increases the risk of perioperative stroke, particularly among patient with atrial fibrillation, therefore preoperative echocardiography may help to stratify the risk of stroke. Atrial fibrillation occurs in 30 to $50 \%$ of patients after cardiac surgery with a peak incidence between the second and fourth postoperative days and it is a major cause of many perioperative strokes.

The incidence of postoperative atrial fibrillation and stroke may be reduced by the prophylactic administration of amiodarone and beta-blockers, beginning 5 days before cardiac surgery.

The discontinuation of warfarin or antiplatelet agents in anticipation of surgery exposes patients to an increased risk of perioperative stroke. The risk is particularly high among patients with coronary artery 
disease. Urgent or emergent catheterization confer an additional risk for CVA.

Possible explanations include the greater propensity for hemodynamic compromise in these patients, which may increase the risk of ischemic stroke and the less meticulous care in the advancement of catheters through the aorta during urgent or emergent PCI, which increases the risk of embolization to the brain. Keely and Grines (1998), in fact, showed that scraping of aortic plaque occurs in more than $50 \%$ percutaneous revascularization procedures.

\section{CONCLUSION}

This report shows that occurrence of periprocedural CVAs related to cardiac catheterization can be observed and that, especially when risk factors are present, careful attention should be devoted to the preparation of the patient and to minimize catheter manipulation and exchanges.

\section{REFERENCES}

Bendszus, M., M. Koltzenburg, R. Burger, M. WarmuthMetz and E. Hofmann et al., 1999. Silent embolism in diagnostic cerebral angiography and neurointerventional procedures: A prospective study. Lancet, 354: 1594-1597. PMID: 10560674

Burdette, J.H., P.E. Ricci, N. Petitti and A.D. Elster, 1998. Cerebral infarction: Time course of signal intensity changes on diffusion-weighted MR images. Am. J. Roentgenol., 171: 791-795. DOI: 10.2214/ajr.171.3.9725318

Büsing, K.A., C. Schulte-Sasse, S. Flüchter and T. Süselbeck, 2005. Cerebral infarction: Incidence and risk factors after diagnostic and interventional cardiac catheterization-prospective evaluation at diffusion-weighted MR imaging. Radiology, 235: 177-183. DOI: 10.1148/radiol.2351040117

Büsing, K.A., C. Schulte-Sasse, S. Flüchter and T. Süselbeck, 2005. Cerebral infarction: Incidence and risk factors after diagnostic and interventional cardiac catheterization-prospective evaluation at diffusion-weighted MR imaging. Radiology, 235: 177-183. DOI: 10.1148/radiol.2351040117

Dukkipati, S., W.W. O’Neill, K.J. Harjai, W.P. Sanders and D. Deo et al., 2004. Characteristics of cerebrovascular accidents after percutaneous coronary intervention. J. Am. Coll. Cardiol., 43: 1161-1167. DOI: 10.1016/j.jacc.2003.11.033
Fiebach, J., O. Jansen, P. Schellinger, M. Knauth and M. Hartmann et al., 2001. Comparison of CT with diffusion-weighted MRI in patients with hyperacute stroke. Neuroradiology, 43: 628-632. DOI: 10.1007/s002340100542

Fuchs, S., E. Stabile, T.D. Kinnaird, G.S. Mintz and L. Gruberg et al., 2002. Stroke complicating percutaneous coronary interventions: Incidence, predictors and prognostic implications. Circulation, 106: 86-91. DOI: 10.1161/01.CIR.0000020678.16325.E0

Hähnel, S., J. Bender, O. Jansen, M. Hartmann and M. Knauth et al., 2001. Clinically silent cerebral embolisms after cerebral catheter angiography. Rofo, 173: 300-305. PMID: 11367837

Jackson, J.L., G.S. Meyer and T. Pettit, 2000. Complications from cardiac catheterization: Analysis of a military database. Mil. Med., 165: 298-301. PMID: 10803005

Jaeger, H.J., K.D. Mathias, R. Drescher, E. Hauth and G. Bockisch et al., 2001. Diffusion-weighted MR imaging after angioplasty or angioplasty plus stenting of arteries supplying the brain. AJNR Am. J. Neuroradiol., 22: 1251-1259. PMID: 11498411

Keely, E.C. and C.L. Grines, 1998. Scraping of aortic debris by coronary guiding catheters: A prospective evaluation of 1,000 cases. J. Am. Coll. Cardiol., 32: 1861-1865. PMID: 9857864

Lund, C., R.B. Nes, T.P. Ugelstad, P. Due-Tønnessen and R. Andersen et al., 2005. Cerebral emboli during left heart catheterization may cause acute brain injury. Eur. Heart J., 26: 1269-1275. DOI: 10.1093/eurheartj/ehi148

Miyazava, N., K. Hashizume, M. Uchida and H. Nukui, 2001. Long-term follow-up of asymptomatic patients with major artery occlusion: Rate of symptomatic change and evaluation of cerebral hemodynamics. Am. J. Neuroradiol., 22: 243-247. PMID: 11156763

Omran, H., H. Schmidt, M. Hackenbroch, S. Illien and P. Bernhardt et al., 2003. Silent and apparent cerebral embolism after retrograde catheterisation of the aortic valve in valvular stenosis: A prospective, randomised study. Lancet, 361: 1241-1246. PMID: 12699950

Weiller, C., E.B. Ringelstein, W. Reiche and U. Buell, 1991. Clinical and hemodynamic aspects of low-flow infarcts. Stroke, 22: 1117-1123. PMID: 1926254

Wityk, R.J., M.A. Goldsborough, A. Hillis, N. Beauchamp and P.B. Barker et al., 2001. Diffusion-and perfusion-weighted brain magnetic resonance imaging in patients with neurologic complications after cardiac surgery. Arch. Neurol., 58: 571-576. PMID: 11295987 